\title{
Autocrine motility factor promotes epithelial-mesenchymal transition in endometrial cancer via MAPK signaling pathway
}

\author{
YIRAN LI $^{1,3}$, QI CHE ${ }^{1}$, YIDING BIAN ${ }^{3}$, QIAN ZHOU ${ }^{2}$, FEIZHOU JIANG ${ }^{1}$, \\ HUAN TONG ${ }^{1}$, JIEQI KE ${ }^{1}$, KAI WANG ${ }^{2}$ and XIAO-PING WAN ${ }^{3}$
}

\begin{abstract}
${ }^{1}$ Department of Obstetrics and Gynecology, Shanghai First People's Hospital Affiliated to Shanghai Jiao Tong University, Shanghai 200040; ${ }^{2}$ Clinical and Translational Research Center, ${ }^{3}$ Department of Gynecology, Shanghai First Maternity and Infant Hospital, Tongji University School of Medicine, Shanghai 200040, P.R. China
\end{abstract}

Received May 27, 2015; Accepted July 9, 2015

DOI: 10.3892/ijo.2015.3091

\begin{abstract}
Autocrine motility factor (AMF) as a cytokine and a growth factor, is known to regulate tumor cell growth and motility in the progress of various human malignant tumors, however, its role in endometrial cancer (EC) has not been fully studied. In the present study, using immunohistochemistry, we found that AMF was highly expressed in EC tissues compared with normal endometrial tissues and tissue micrioarray technology showed positive correlation between AMF expression and epithelial-to-mesenchymal transition (EMT) related markers E-cadherin, vimentin and Snail. Next, we detected that silencing of AMF by stable transfection with shRNA induced mesenchymal-to-epithelial transition phenotype in Ishikawa and HEC-1B cells by qRT-PCR, western blotting and immunofluorescence. Gene expression profile revealed that AMF silencing resulted in altered expression of EMT related molecular mediators including Snail and transforming growth factor $\beta$ receptor 1 , and involvement of mitogen-activated protein kinase (MAPK) signaling pathway. Additionally, we found that EMT related markers were downregulated with pretreatment of the MAPK-specific inhibitor U0126 by western blotting. The present study is the first to support a role for AMF mediating EMT in endometrial cancer through MAPK signaling. Therefore, AMF may provide a potential prognostic and therapeutic target in preventing EC progression.
\end{abstract}

Correspondence to: Dr Kai Wang, Clinical and Translational Research Center, Shanghai First Maternity and Infant Hospital, Tongji University School of Medicine, 536 Changle Road, Jingan, Shanghai 200040, P.R. China

E-mail: kaiwangcn@yahoo.com

Professor Xiao-Ping Wan, Department of Gynecology, Shanghai First Maternity and Infant Hospital, Tongji University School of Medicine, 536 Changle Road, Jingan, Shanghai 200040, P.R. China E-mail:wanxp@sjtu.edu.cn

Key words: autocrine motility factor, endometrial cancer, epithelialmesenchymal transition, mesenchymal-epithelial transition, mitogenactivated protein kinase signaling pathway

\section{Introduction}

Endometrial cancer (EC) is the most common gynecological malignancy of the female genital tract. For 2015, it is estimated that $\sim 54,870$ new cancer cases of EC will be diagnosed and 10,170 deaths are expected in the USA alone (1). Although most EC is diagnosed at early-stage which correlates with advanced outcome, women with late-stage EC are at highest risk of recurrence and poor prognosis $(2,3)$. In particular, EC cases with regional or distal metastasis recur with limited effect of systemic therapies (2). It is, therefore, important to further elucidate the molecular and cellular mechanisms responsible for tumorigenesis and progression of $\mathrm{EC}$, and to develop novel diagnostic and therapeutic strategies.

Autocrine motility factor (AMF), which is also known as phosphoglucose isomerase (PGI), serves as tumor-secreted cytokine, it stimulates tumor cells motility, migration, invasion and metastasis $(4,5)$. AMF/PGI also behaves as a housekeeping cytosolic enzyme of sugar metabolism and plays a key role during glycolysis and gluconeogenesis, catalyzing the interconversion of glucose 6-phosphate and fructose 6-phosphate (6). In addition, AMF/PGI serves several other functions as neuroleukin, which promotes growth of embryonic spinal and sensory neurons (7); and as a maturation factor mediating differentiation of human myeloid leukemia cells (8). Several researchers independently found that secreted AMF/PGI by tumor cells is involved in regulation of oncogenesis and tumor progression in various human cancers (9-13).

Recent studies have shown that PGI/AMF plays an important role during epithelial-to-mesenchymal transition (EMT) which is an essential mechanism for the development of malignant tumors for invasion and metastasis (13-15). EMT was well-documented as the process that produces a complete loss of epithelial traits by the former epithelial cells accompanied by the acquisition of mesenchymal characteristics in vitro $(16,17)$ and was reflected in changes from a tightly organized cobblestone-like structure, whereby the cells adhere to each other and manifest apical basal polarity to spindly migratory elongated cells with disrupted cell-cell/cell-substratum contacts $(15,18)$. The phenomenon of EMT is associated with acquisition of invasive phenotype by cancer cells $(19,20)$ and, 
in particular, aggressive behavior of endometrial cancer (21). The role of PGI/AMF during EMT has been extensively described in other types of cancer but has been poorly studied in EC $(22,23)$. Therefore, it is of considerable interest to identify novel mechanisms to better understand how EC occurs and how it is disseminated.

In the present report, we describe that the association between expression of AMF and EMT in EC specimens, and we also identified a critical role of $\mathrm{AMF} / \mathrm{PGI}$ in promoting EMT in EC. These results shed light on the mechanisms by which EC occurs and develops and provide evidence that AMF/PGI as a novel proto-oncoprotein of EC and therefore a potential therapeutic target.

\section{Materials and methods}

Reagents and antibodies. Purified rabbit AMF was purchased from Sigma for exogenous AMF stimulation (cat. no P9544). Mouse monoclonal anti-AMF (ab66340), rabbit polyclonal anti-AMF (ab86950), E-cadherin (ab15148), vimentin (ab45939), Snail (ab180714), TGFBR1 (abab31013) and anti$\beta$-actin (ab8227) for use in immunohistochemistry or western blot analyses were obtained from Abcam Ltd. (Hong Kong, China). U0126 (MAPK inhibitor) was purchased from Selleck Chemicals.

Cell culture. Ishikawa and HEC-1B cells were purchased from the American Type Culture Collection (ATCC; Manassas, VA, USA) and maintained according to the provider's instruction in DMEM/F12 media (Gibco, Auckland, New Zealand) supplemented with $10 \%$ fetal bovine serum (FBS; Gibco, Carlsbad, CA, USA). All cells were grown until confluent and were incubated in serum-free medium for $24 \mathrm{~h}$ before treatment with various experimental agents as described.

Tissue microarray slides and immunohistochemistry. High-density tissue microarrays were constructed by Outdo Biotech Co., Ltd., (Shanghai, China) using clinical samples obtained from a cohort of 62 patients who underwent surgery at the Shanghai First People's Hospital. Each array includes 31 normal and 31 malignant tissue specimens of the endometrium (various grades and stages). Paraffin-embedded tissue sections $(5 \mu \mathrm{m})$ were deparaffinized by xylene and rehydrated in a graded alcohol series $(100,95,80$ and $70 \%$, 5 min each). Antigen retrieval was done by boiling the slides in a small beaker filled with $1 \mathrm{mM}$ EDTA ( $\mathrm{pH} 8.0$ ). Endogenous peroxidase activity was quenched by a $10-\mathrm{min}$ incubation in $3 \%$ hydrogen peroxide. After antigen retrieval, the slides were washed two times in a $0.1 \%$ Tween/1X TBS $(0.1 \%$ TBST) bath $(5 \mathrm{~min}$ each) at room temperature to remove non-specific background binding. Protein blocking was performed by incubating the specimens in $5 \%$ normal rabbit serum or normal horse serum in $0.1 \%$ TBS for $1 \mathrm{~h}$. Primary antibodies against AMF, E-cadherin or Snail and vimentin were diluted 1:200 and 1:500, respectively and applied for $1 \mathrm{~h}$ at room temperature. After a series of TBST rinses as described above, bound antibody was subsequently detected using EnVision reagents (Wuhan Boster Biological Engineering Co., Ltd., Wuhan, China) according to the manufacturer's instructions.
Evaluation of immunohistochemical staining and statistical analysis. Immunostained slides were scored under a microscope. The staining intensity was scored as 0 (negative), 1 (weak), 2 (medium) or 3 (strong). The extent of staining was scored as $0(0 \%), 1(1-25 \%), 2(26-50 \%), 3(51-75 \%)$ or $4(76-100 \%)$, according to the percentage of the positively stained areas in relation to the whole tumor area. The sum of the intensity score and the extent score was used as the final staining score (0-7). Tumors with a final staining score of 4 or higher were considered 'positive' (24). Results were assessed by two pathologists in a blinded manner with respect to the tissue source.

Stable silencing of AMF expression by short hairpin RNA (shRNA). AMF shRNA constructs were cloned into pLKO.1 plasmid under the control of U6 promoter for stable expression (Sigma). Three pairs of annealed DNA oligonucleotides were inserted into the AgeI and EcoRI restriction sites of pLKO.1. The most effective pairs of sequence targeted to human AMF is: sense, 5'-CGCCATGTATGAGCACAAGAT-3' and antisense 5'-GCGGTACATACTCGTGTTCTA-3' (shAMF-1), as well as sense 5'-CCTGTCTACTAACACAACCAA-3' and antisense 5'-GGACAGATGATTGTGTTGGTT-3' (shAMF-2), respectively. Ishikawa and HEC-1B cells were infected with lentiviral vector pLKO.1 (mock) or AMF-specific shRNA lentiviral particles in 6-well plates in the presence of polybrene $(6 \mathrm{mg} / \mathrm{ml})$ and then treated with puromycin $(2 \mathrm{mg} / \mathrm{ml})$ to generate stable clones. Puromycin-resistant AMF knockdown clones were harvested by ring selection, and AMF gene expression and protein level were confirmed by qRT-PCR and immunoblotting.

RNA extraction and $q R T-P C R$ (quantitative real-time polymerase chain reaction). Total RNA was isolated using TRIzol reagent (Invitrogen, Life Technologies; Shanghai, China) and reverse transcribed using a reverse transcriptase kit (Takara, Dalian, China). Gene expression was detected with SYBRGreen Master Mix (Takara) on an ABI Prism 700 Thermal Cycler (Applied Biosystems, Foster City, CA, USA). Gene expression was calculated using the $2^{-\Delta \Delta \mathrm{Ct}}$ formula and normalized against $\beta$-actin. The oligonucleotide primers were 5'-CGCCCAACCAACTCTATTG-3' (forward) and 5'-GAT GATGCCCTGAACGAAG-3' (reverse) for human AMF detection; 5'-TTATGATTCTCTGCTCGTG-3' (forward) and 5'-ATAGTCCTGGTCTTTGTCT-3' (reverse) for human E-cadherin detection; 5'-AGGAGGAAATGGCTCGTCA-3' (forward) and 5'-TGTAGGTGGCAATCTCAAT-3' (reverse) for human vimentin detection; 5'-CGGCTCCTTCGTCCT TC-3' (forward) and 5'-GCACCCAGGCTGAGGTATT-3' (reverse) for human Snail detection; 5'-TGTGAAGCCTTGAG AGTAA-3' (forward) and 5'-TGTTGACTGAGTTGCGATA-3' (reverse) for human TGFBR1 detection; 5'-CAGCCATGT ACGTTGCTATCCAGG-3' (forward) and 5'-AGGTCCAGA CGCAGGATGGCATG-3' (reverse) for human $\beta$-actin detection (as a housekeeping gene). All experiments were performed independently in triplicate.

Protein extraction and western blot analysis. For whole-cell lysates, cells were washed twice with PBS and collected by scraping. Cell pellets were lysed in cold precipitation assay 
buffer [20 mM Tris-HCl (pH 7.4), $150 \mathrm{mM} \mathrm{NaCl}, 10 \mathrm{mM}$ EDTA, 1\% NP40, Triton X-100, sodium deoxycholate] containing $1 \mathrm{mmol} / \mathrm{l} \mathrm{DTT}, 1 \mathrm{mmol} / 1$ phenylmethylsulfonyl fluoride, $10 \mu \mathrm{g} / \mathrm{ml}$ leupeptin and $10 \mu \mathrm{g} / \mathrm{ml}$ aprotinin. Samples were separated by centrifugation $\left(15,000 \mathrm{rpm}\right.$ in $4^{\circ} \mathrm{C}$ for $30 \mathrm{~min})$. Lysate supernatants were 100 -fold concentrated with Amicon Ultra (30,000 nominal molecular weight limit; Millipore).

The extracted protein concentration was measured with BCA Protein assay kit (Thermo Fisher Scientific). Equal amounts of the proteins $(20 \mu \mathrm{g}$ of cellular protein or $80 \mu \mathrm{g}$ of secreted protein) were separated on $10 \%$ SDS-PAGE gels and transferred to 0.2-Am PVDF membrane (Osmonics, Inc.). Each membrane was then incubated overnight at $4^{\circ} \mathrm{C}$ with an appropriate diluted primary antibody. HRP-secondary anti-rabbit or anti-mouse antibodies (diluted 1:5,000 to yield $0.2 \mathrm{mg} / \mathrm{ml}$; Abcam) were used to detect the bound primary antibodies. Blots were visualized using the Bioscience Odyssey Infrared Imaging System (LI-COR Biosciences), and band density was quantitated using ImageJ imaging analysis software (NIH, Bethesda, MD, USA). Data were normalized to $\beta$-actin expression by densitometry and statistical data from at least three experiments were recorded.

Immunofluorescence and imaging. For detection of E-cadherin and vimentin, cells were seeded onto glass coverslips, fixed with $4 \%$ paraformaldehyde for $10 \mathrm{~min}$ and permeabilized with PBS Triton X-100 4\% for 10 min. Cells were sequentially incubated with primary antibody (rabbit anti-E-cadherin and anti-vimentin 1:200) diluted in blocking buffer overnight at $4^{\circ} \mathrm{C}$, which was followed by incubation with secondary antibodies conjugated with Alexa Fluor 568 (Molecular Probes-Invitrogen, Carlsbad, CA, USA) for $1 \mathrm{~h}$ at RT in the dark. After washing with PBS, the cells were stained with 4',6'-diamidino-2-phenylindole (DAPI; targeting DNA in the cell nucleus) for $5 \mathrm{~min}$. After extensive washing, the cells were mounted on a glass slide with $80 \%$ glycerol and fluorescent images were analyzed in an Olympus fluorescence microscope using a x400 lens.

Microarray and data analysis. The Agilent SurePrint G3 Human Gene Expression microarray $(8 \times 60 \mathrm{~K})$ was used in the present study. This chip targets $>30,000$ genes with $>50,000$ probes and $>12,000$ lincRNA derived from a broad survey of well known sources such as RefSeq, Ensembl, UniGene and others. The resulting view of the human genome covers $30 \mathrm{~K}$ unique genes and transcripts that have been verified and optimized by alignment to the human genome assembly and by the Agilent Empirical Validation process. Total RNA (>300 ng) was extracted from four independent cultures of Ishikawa, HEC-1B cells, shAMF-1 (Ishikawa) and shAMF-1 (HEC-1B) cells. Microarray hybridization, data collection and analysis were performed at Oebiotech Biotechnology Corp. (Shanghai, China). The threshold set for upregulated and downregulated genes was a fold-change $\geq 2.0$. KEGG analysis was applied to determine the roles of these differentially expressed mRNAs.

U0126 and exogenous PGI/AMF treatment. Cells were seeded at $1.0 \times 10^{5}$ cells/well of a 12 -well plate, containing $1.2 \mathrm{ml}$ regular medium. The media was then changed to phenol-red free medium with $0.5 \%$ stripped FBS for incubation at $37^{\circ} \mathrm{C}$ overnight. Immediately prior to treatment, the medium in the culture plates was aspirated, triply washed with PBS and replaced with fresh medium. U0126 dissolved in DMSO or PGI/AMF dissolved in PBS was subsequently added to each well. Concurrently, the same amount of DMSO or PBS was added to the control wells.

Statistical analysis. Continuous variables were recorded as mean \pm SD and all statistical analyses were done using Statistical Package for the Social Sciences (SPSS) software version 17.0 (SPSS, Inc., Chicago, IL, USA). Volumetric data were assessed using an unpaired Student's t-test, or one-way ANOVA analysis followed by post-hoc LSD test or Dunnett's test for multiple comparisons. Concordance and correlation among antibodies were accessed by calculating Chi-square test and McNemar's statistical test. P-values $<0.05$ were considered statistically significant. All experiments were repeated independently at least three times.

\section{Results}

Autocrine motility factor was highly expressed and correlate with EMT in EC tissues (patients clinical information). AMF abnormal expression has been associated with tumor progression and EMT phenotype conversions in many human cancers $(13,22,23)$. In our studies, immunochemistry staining showed that AMF protein was predominantly localized to the cytoplasm of endometrial epithelial cells. There was only weak or no staining in normal endometrium, whereas strong AMF immunostaining was found in endometrial carcinoma tissues, including endometrioid adenocarcinoma, uterine papillary serous carcinoma and endometrial clear cell carcinoma (Fig. 1A). The mean scores for AMF staining were 4.5 and 0.95 for cancer tissue and normal human endometrium, respectively $(\mathrm{P}<0.001)$ (Fig. 1B). Furthermore, we used a semi-quantitative analysis of IHC staining to evaluate AMF and EMT markers including E-cadherin, vimentin and Snail expression in the tissues microarrays. AMF levels positively correlated with vimentin $(\mathrm{P}=0.012)$ and Snail $(\mathrm{P}=0.021)$ levels, inversely correlated with the levels of E-cadherin $(\mathrm{P}=0.035)$ (Table I). These data indicated that AMF expression was much higher in EC tissue specimens and relevant to the levels of EMT marker protein.

Silencing of AMF reverses the EMT phenotype in EC cells. Processes involved in the EMT are closely correlated with cancer metastasis. The expression of AMF and EMT markers in EC tissue specimens prompted us to examine whether AMF silencing could induce morphologic changes, loss of mesenchymal and/or gain of epithelial markers. The human EC cell lines Ishikawa and HEC-1B were stably expressed with either shAMF or control vectors. We microscopically examined AMF-silencing EC cells to determine the effects of AMF on cellular morphology. Ishikawa ${ }^{\text {shAMF }}$ and HEC-1B ${ }^{\text {shAMF }}$ cells were morphologically transformed toward epithelia compared with Ishikawa, HEC-1B and their control cells (Fig. 2A). This change was characterized a transition from the spindle-like morphology of the parental cells to a more differentiated keratinocyte-like morphology, suggesting a phenotypic transi- 
A

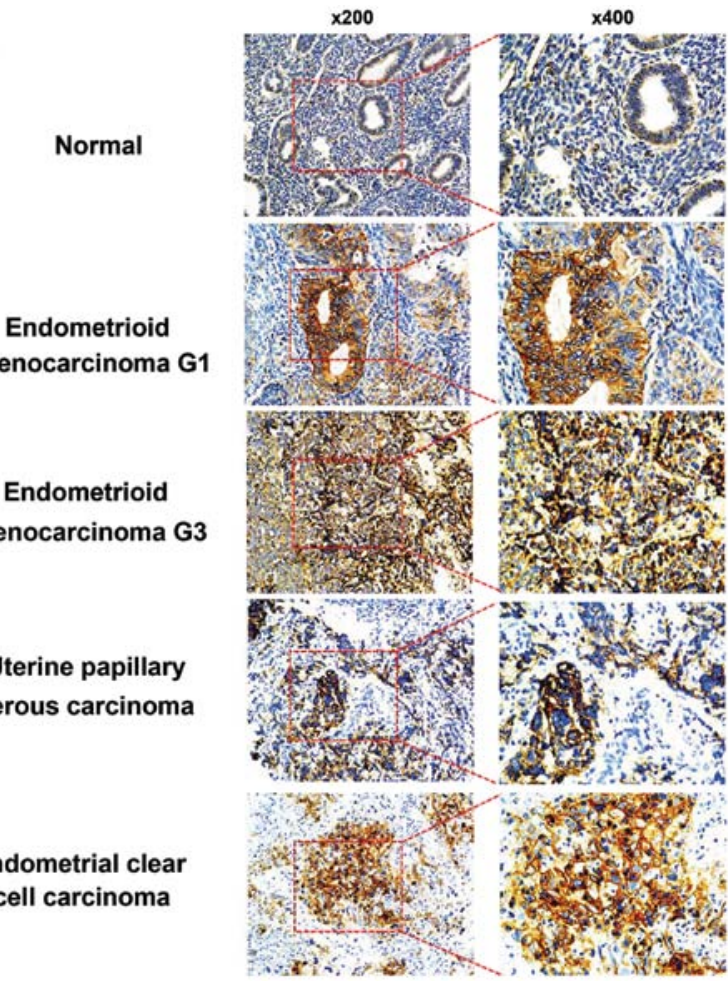

B
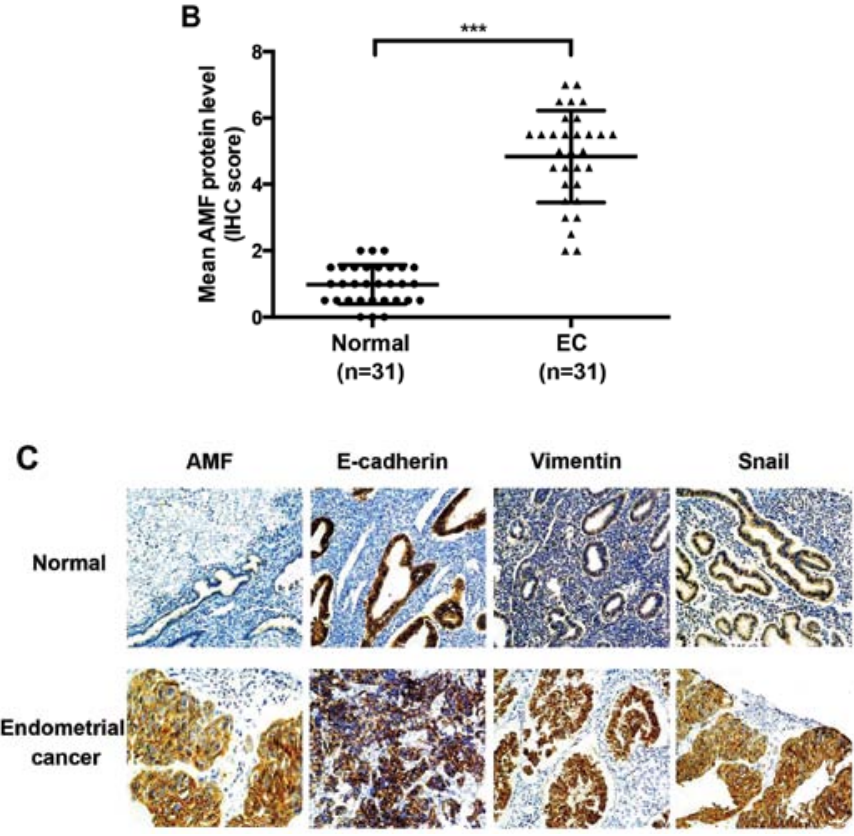

Figure 1. The expression of AMF and EMT markers in tissues microarrays. (A) Immunohistochemical analysis of AMF expression in normal endometrium, endometrioid adenocarcinoma G1/G3, uterine papillary serous carcinoma and endometrial clear cell carcinoma (left line, x200; right line, $\mathrm{x} 400$ ). No or weak expression of AMF in normal endometrium, strong cytoplasm and cell membrane expression of AMF in the majority of tumor. (B) Results of immunostaining score in normal endometrium and EC $\left({ }^{* * *} \mathrm{P}<0.001\right)$. (C) Expression of AMF, E-cadherin, vimentin and Snail in normal tissues and endometrial cancer tissues.

tion from mesenchymal to epithelial. Thus, the expression of the intermediate filaments vimentin (a mesenchymal marker) and E-cadherin (an epithelial marker) was examined by immunofluorescent staining (Fig. 2B). Vimentin was prominently expressed throughout the cytoplasm of the parental Ishikawa and HEC-1B cells, whereas a significantly weaker vimentin signal was detected in their shAMF cell clone. Next, we tested whether the reduced vimentin expression was associated with a gain of epithelial markers. The cells were immunostained for the presence of the mesenchymal intermediate filament marker (i.e., E-cadherin). Either the parental Ishikawa or HEC-1B cells expressed E-cadherin proteins at a low level, while E-cadherin expression increased in the shAMF cells (Fig. 2B).

To further determine if this transformation represented an EMT [as has been reported (23)], we analyzed the levels of AMF and several characteristic epithelial and mesenchymal proteins by qRT-PCR and western blotting. We observed the expression of AMF was markedly decreased by stable transfection with shAMF (Fig. 2C and D). Furthermore, the levels of vimentin expression were reduced, while E-cadherin expression levels were raised in Ishikawa ${ }^{\text {shAMF }}$ and HEC-1B ${ }^{\text {shAMF }}$ cells (Fig. 2C and D). Taken together, these findings indicate that AMF silencing is accompanied by the loss of mesenchymal and the gain of epithelial markers, and show that AMF likely plays a crucial role in the regulation of the EMT in EC cells.

Identification of downregulated genes associated with EMT in AMF silencing cell lines. To investigate the functional significance of AMF involved in EMT in EC cells, we compared the expression profiles of the gene associated with EMT in Ishikawa/Ishikawa ${ }^{\text {shAMF }}$ and HEC-1B/HEC-1B ${ }^{\text {shAMF}}$. Pairwise comparisons identified 46 differentially expressed genes that had changed by at least 2 -fold after AMF silencing in Ishikawa. In contrast, only 34 differentially expressed genes were identified following AMF silencing of HEC-1B. We generated Venn diagrams by combining the separate gene lists obtained from the GeneSifter analysis, and then manually identified the common 15 genes that were regulated by AMF silencing in both Ishikawa and HEC-1B (Fig. 3A). To validate the array data, we performed qRT-PCR for two genes: Snail and transforming growth factor $\beta$ receptor 1 (TGFBR1) whose expression was significantly downregulated by AMF silencing in both Ishikawa and HEC-1B. A comparison of the fold induction of each of the two genes as determined by microarray and qRT-PCR found that induction of gene expression as determined by qRT-PCR was consistently with that determined by microarrays (Fig. 3B). We further confirmed by western blotting that AMF silencing markedly downregulated the expression of the two genes in both Ishikawa and HEC-1B (Fig. 3C). These results demonstrated that AMF activated Snail and TGFBR1 regulated EMT in Ishikawa and HEC-1B cells.

AMF mediated EMT by activating MAPK pathway in EC cells. To explore which pathways were activated during EMT mediated by AMF, we categorized the 15 differentially expressed genes commonly regulated by AMF silencing in both Ishikawa and HEC-1B using the KEGG pathway analysis tool provided in GeneSifter software. As shown in Fig. 4A, the total network comprised 9 distinct KEGG pathways, including pathways related to EMT (mitogen-activated protein kinase pathway and focal adhesion pathway) $(19,20)$. In addition, two of these 15 differentially expressed genes were previously verified downregulated in AMF silencing cells including 
A
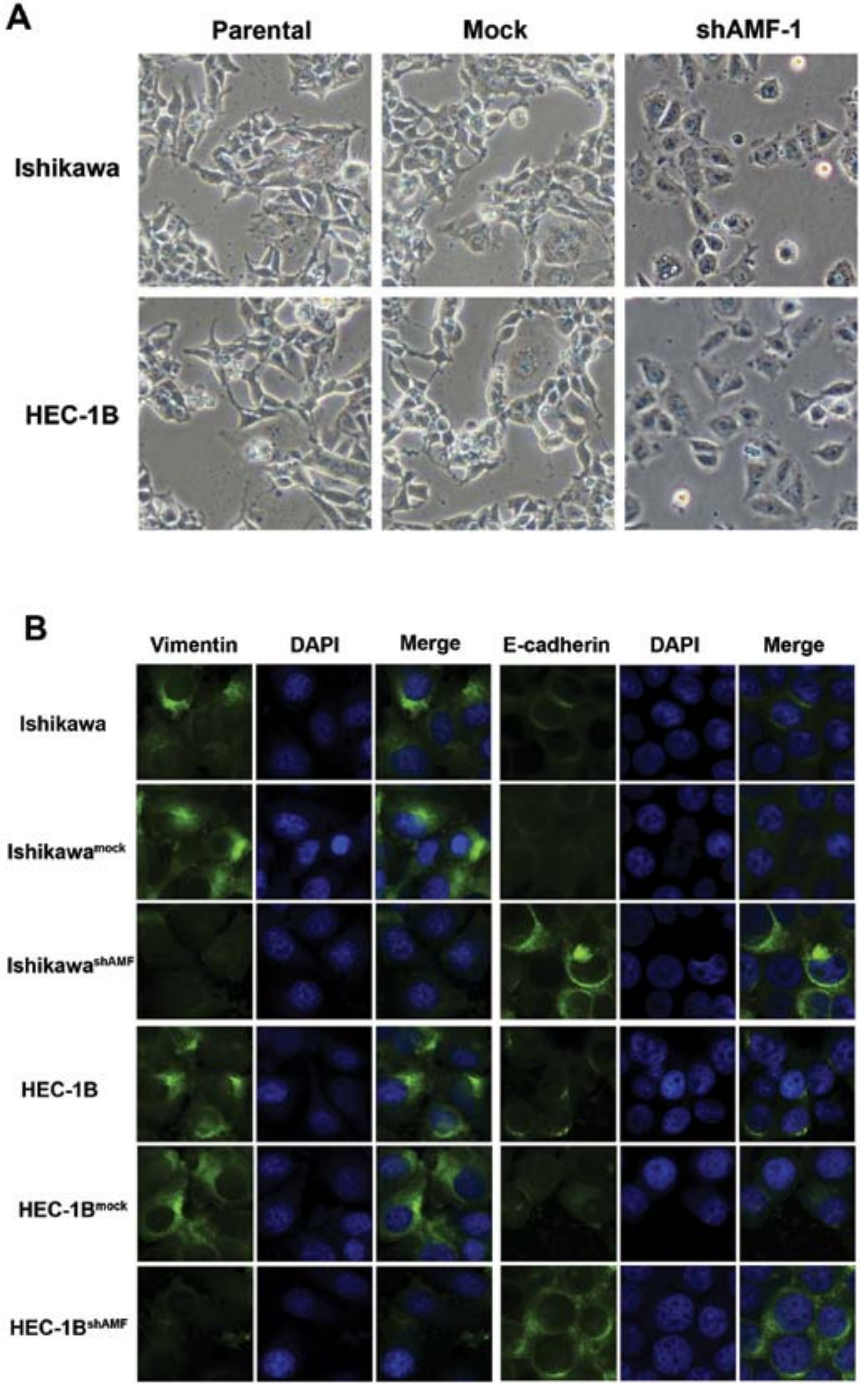
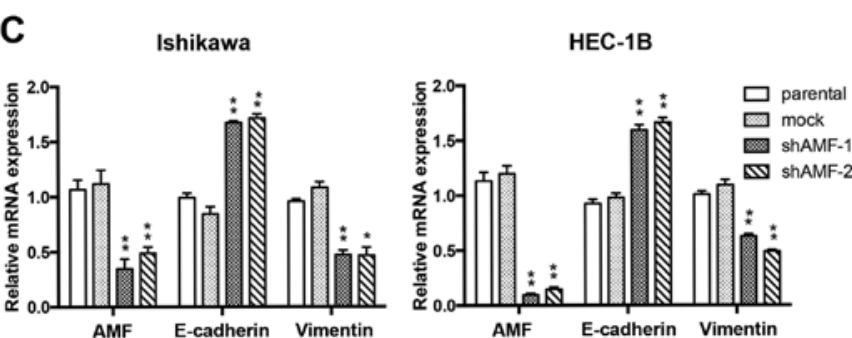

D
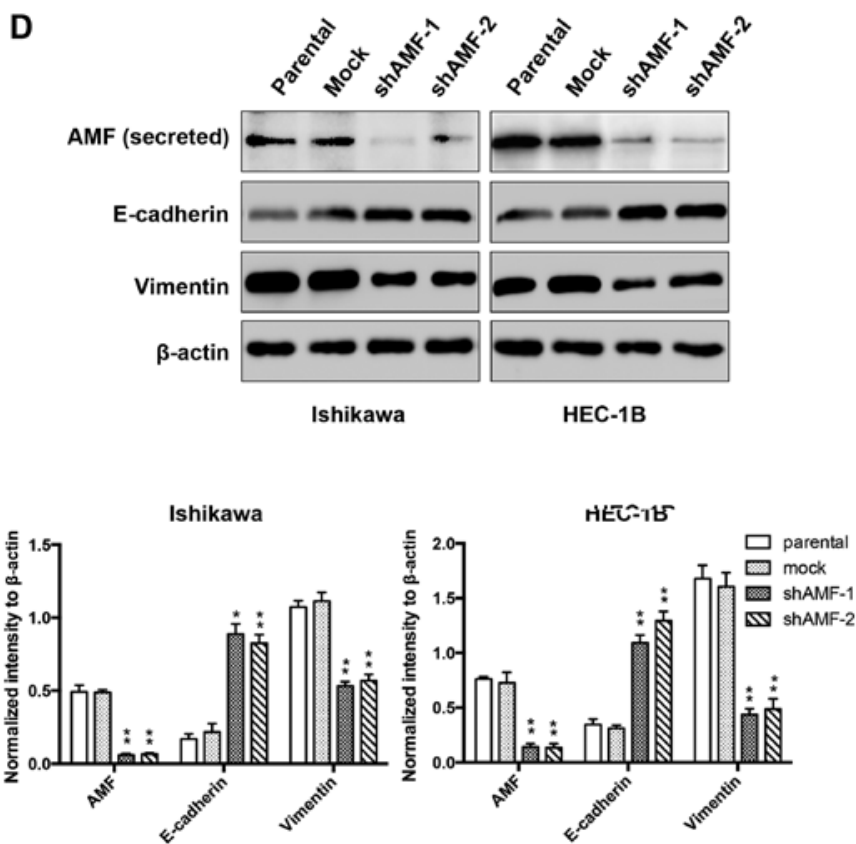

Figure 2. Silencing of AMF reverses the EMT phenotype in EC cells. (A) Epithelial morphology induced by suppression of AMF in Ishikawa and HEC-1B cells (magnification, x200). (B) Immunofluorescence analysis. Mesenchymal marker vimentin and epithelial marker E-cadherin were visualized by immunofluorescence (green) following staining for nucleus with DAPI (blue), (magnification, x400). (C) mRNA levels of AMF and EMT markers as analyzed by qRT-PCR in Ishikawa and HEC-1B cell lines. $\beta$-actin was included as an internal control. (D) Protein levels of AMF and EMT markers in Ishikawa and HEC-1B cells as analyzed by immunoblotting (upper panel), and further quantified by densitometry of triplicate experiments (lower panel). $\beta$-actin was included as an internal control. $\left({ }^{*} \mathrm{P}<0.05 ;{ }^{* * *} \mathrm{P}<0.01\right)$.

Table I. Expression correlations between AMF and E-cadherin, vimentin and Snail.

AMF*E-cadherin/Vimentin/Snail

\begin{tabular}{|c|c|c|c|c|}
\hline E-cadherin & \multicolumn{2}{|c|}{ Vimentin } & \multicolumn{2}{|c|}{ Snail } \\
\hline+ & - & + & - & + \\
\hline
\end{tabular}

\begin{tabular}{clllllll}
\hline AMF & & & & & & & \\
- & Count & 4 & 6 & 9 & 1 & 9 & 1 \\
& \% of Total & $13 \%$ & $19 \%$ & $29 \%$ & $3 \%$ & $29 \%$ & $3 \%$ \\
+ & Count & 17 & 4 & 10 & 11 & 9 & 12 \\
& \% of Total & $55 \%$ & $13 \%$ & $32 \%$ & $35 \%$ & $29 \%$ & $39 \%$ \\
Total & Count & 21 & 10 & 19 & 12 & 18 & 13 \\
& \% of Total & $68 \%$ & $32 \%$ & $61 \%$ & $39 \%$ & $58 \%$ & $42 \%$
\end{tabular}

Statistical analyses were performed using the Chi-square test and McNemar's statistical test. 
A

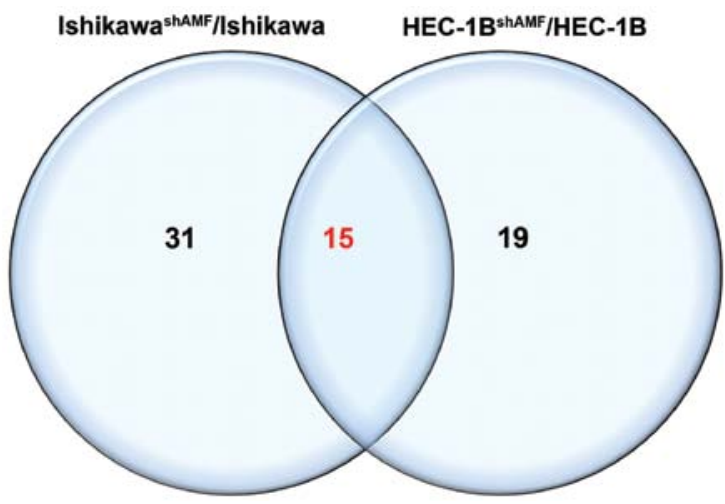

B
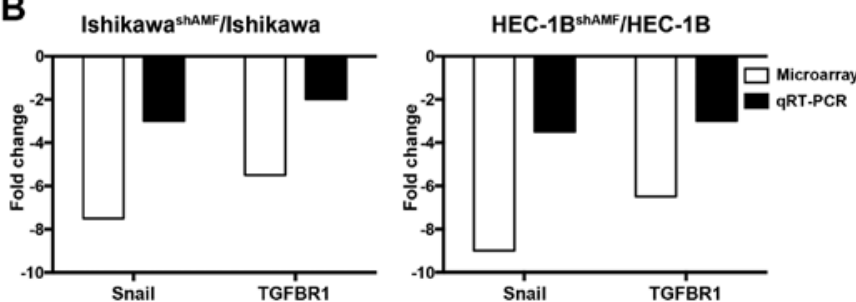

C
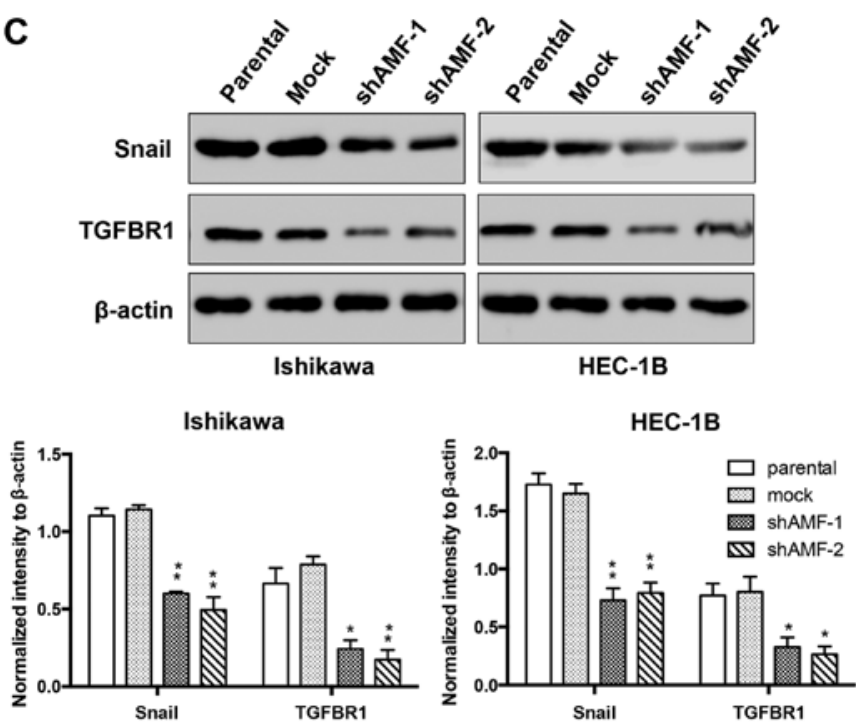

Figure 3. Downregulation of genes associated with EMT in Ishikawa ${ }^{\text {shAMF/ }}$ HEC-1B ${ }^{\text {shAMF }}$ cells. (A) Venn diagrams showing how many genes are regulated ( $\geq 2$-fold) in all cell lines after AMF silencing, and how many of the regulated genes are common between Ishikawa ${ }^{\text {shAMF }}$ and HEC-1B ${ }^{\text {shAMF }}$ (B) Fold-change induced by AMF silencing of the expression of genes, determined by microarray analysis (open bars) and qRT-PCR (solid bars) in Ishikawa ${ }^{\text {shAMF }} /$ Ishikawa and HEC-1B ${ }^{\text {shAMF}} /$ HEC-1B. (C) Protein levels of genes in Ishikawa and HEC-1B cells as analyzed by immunoblotting (upper panel), and further quantified by densitometry of triplicate experiments (lower panel). $\beta$-actin was included as an internal control; $\left({ }^{*} \mathrm{P}<0.05 ;{ }^{* *} \mathrm{P}<0.01\right)$.

Snail and TGFBR1, reside in the MAPK pathway (25). We therefore assessed levels of phosphorylation of the MAPK pathway proteins ERK1/2 (at Thr202/Tyr204) in Ishikawa and HEC-1B cells. Interestingly, the phosphorylation levels of ERK were substantially inhibited by AMF silencing, whereas, exogenous PGI/AMF increased the phosphorylation levels of ERK in AMF silenced cells (Fig. 4B). To determine whether AMF-mediated regulation of the EMT phenotype resulted from the AMF ability to activate the MAPK pathway,

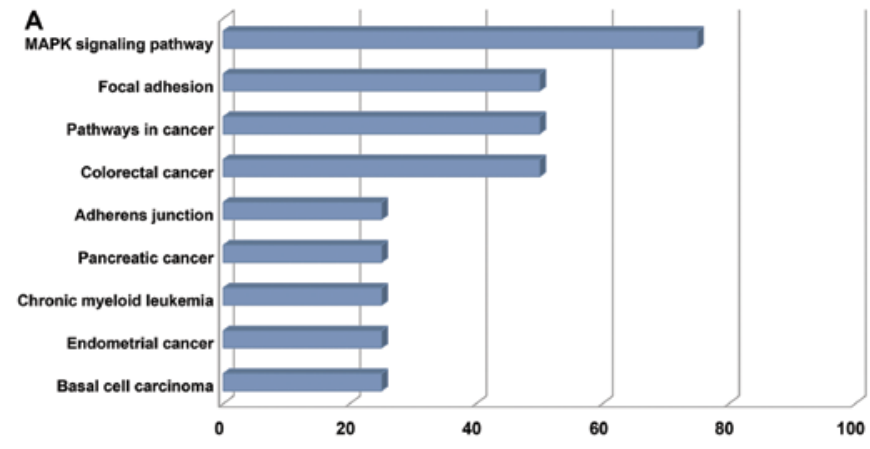

Transcriptional domain coverage (\%)
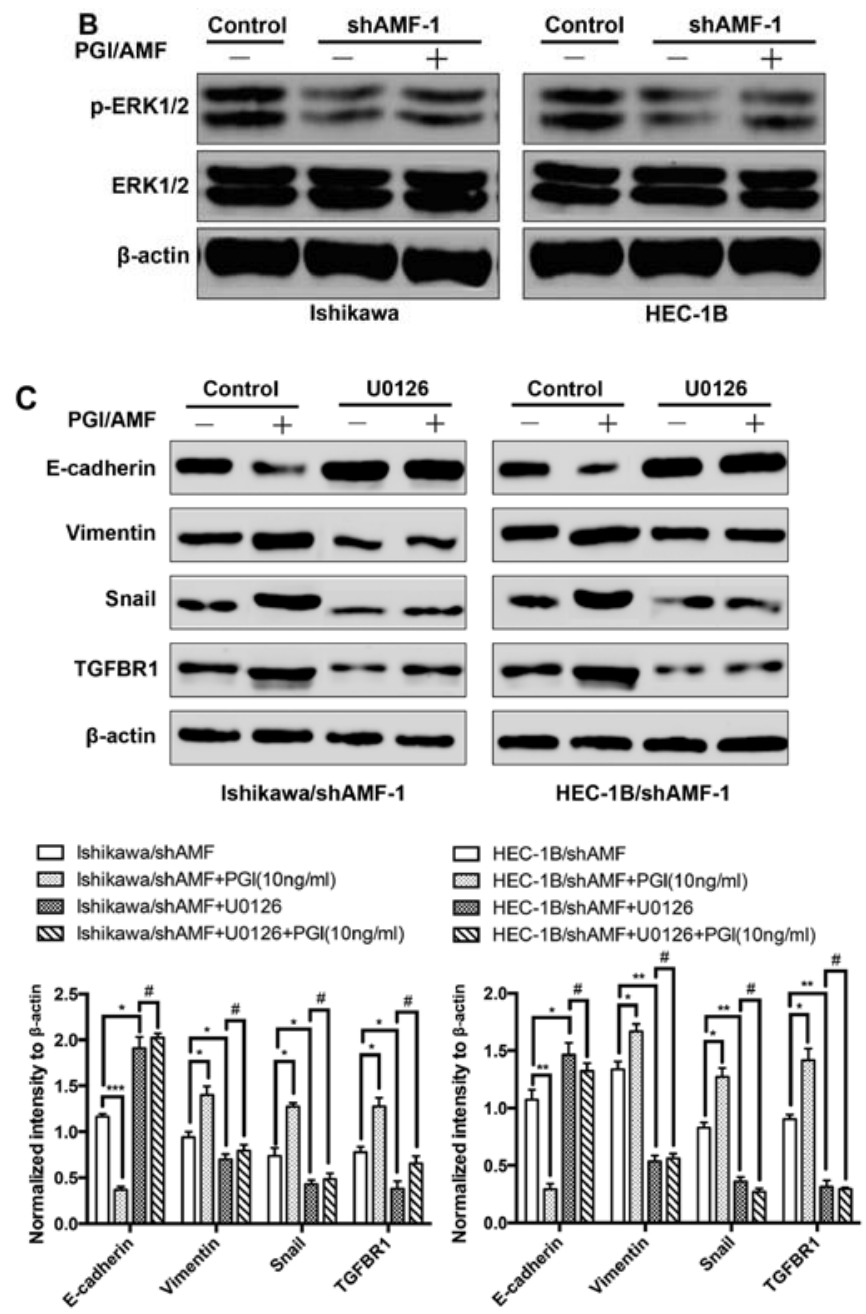

Figure 4. AMF silencing induces MET phenotype through the MAPK pathway in EC cells. (A) Summary of KEGG analysis results for genes belonging to MAPK pathway found markedly changed AMF silencing in Ishikawa and HEC-1B. (B) The effects of AMF/PGI on MAPK signaling in Ishikawa and HEC-1B cells by western blot analysis. $\beta$-actin was included as an internal control. (C) The effect of U0126 (20 $\mu \mathrm{mol} / 1$; MAPK inhibitor) on PGI (10 ng/ml) induced EMT assessed by western blotting (upper panel), and further quantified by densitometry of triplicate experiments (lower panel). $\beta$-actin was included as an internal control. $\left({ }^{*} \mathrm{P}<0.05 ;{ }^{* *} \mathrm{P}<0.01 ;{ }^{* * *} \mathrm{P}<0.001\right.$; "No significant).

U0126 (an inhibitor of MAPK pathway) was used to pretreat Ishikawa $^{\text {shAMF }}$ and HEC-1 $\mathrm{B}^{\text {shAMF }}$ cells. In Ishikawa ${ }^{\text {shAMF }}$ and HEC-1B ${ }^{\text {shAMF }}$ cells, exogenous PGI/AMF decreased the level of the epithelial marker E-cadherin, whereas not the levels of 
the mesenchymal marker vimentin. The EMT inducers Snail and TGFBR1 known as TGF $\beta$ receptor type I $(15,26)$ were upregulated, compared with control groups (Fig. 4C and D). In contrast, the effects of exogenous PGI/AMF on enhancing EMT phenotype were eliminated in U0126 pretreated groups which MAPK pathway was inhibited (Fig. 4C and D). Our findings suggested that MAPK pathway activated by MAPK induced EMT in EC cells.

\section{Discussion}

Tumor metastasis and dissemination are multistep processes involving complex and highly coordinated interactions between tumor cells and a constantly changing host microenvironment (27). Like many other malignant diseases, cancer cells that disseminate from the primary tumor and invade distant organs are the leading causes of death in EC. Secreted AMF/PGI functions as a growth factor as well as a motility factor (28). However, recent work has shown its oncogenic role in tumors, including its ability to regulated EMT $(13,22)$. In the present study, we observed the correlation between AMF and EMT markers in human EC specimens using tissue microarray. Specific silencing of AMF in endometrial cancer cell lines led to changes in tumor cell morphology and the level of EMT marker expression. Gene expression profile analysis investigated the mechanism of regulating EMT by AMF, including gene and signaling pathways.

PGI is essential for an individual cell to survive as the second enzyme in the glycolytic pathway, which seems to be a minimum requirement for the cell, than to secret $\mathrm{AMF}$ as an extracellular form of AMF/PGI. PGI is observed in all cells ubiquitously, whereas secretion of AMF is observed in only tumor cells or activated T cells (5). The extracellular form of AMF, as a growth factor, causes tumor cells to migrate and seems to be involved in tumor invasion and metastasis. Thus, exogenous AMF plays an important role in tumor progression.

Although several studies have explored the oncogenic role of AMF in solid cancers including its ability to regulate tumor aggressiveness $(2,9,14)$, this is the first study to address the function of AMF in human endometrial cancer. As shown in Fig. 1A and B, the normal endometrium produced weak levels of AMF while AMF was extensively detected in endometrial cancer. A correlation between AMF and EMT marker expression was also found in endometrial cancer (Fig. 1C), which was in accordance with reports that AMF induces EMT (13). The EMT plays an important role during cancer progression, leading to a more invasive, metastatic phenotype in human cancers, including EC $(29,30)$. In this process, reduction of E-cadherin expression is required to lose epithelial cell-cell adhesion and to destabilize the epithelial architecture (31). Differentiated mesenchymal cells can spread into tissues surrounding the original tumor as well as separate from the tumor to new locations where they divide and form additional tumors. Our results of the present study confirmed the former hypothesis that AMF silencing resulted in marked changes in cell morphology, reduction of mesenchymal cell marker proteins and detection of epithelial cell marker proteins (32). AMF silencing induced changes of morphology in EC cells, it formed a mesenchymal phenotype with elongated cell shape losing apical basal polarity to the epithelial phenotype which was bound together tightly and exhibiting polarity (Fig. 2A). Furthermore, we observed that the expression of E-cadherin was increased in AMF silenced EC cells with a concomitant decrease in vimentin expression (Fig. 2B and C). These results may suggest that AMF can regulate, in part, epithelial-to-mesenchymal transition during endometrial cancer development.

Gene expression profiles suggested that silencing of AMF in Ishikawa and HEC-1B cells resulted in downregulation of Snail and TGFBR1 (Fig. 3A). Snail, a transcription factor, has been described as a direct repressor of E-cadherin expression during EMT and carcinogenesis $(26,33)$. Phosphorylation of TGFBR1, the type I receptor binding to TGF $\beta$, induced nuclear localization and transcriptional activity of SMADs during TGF $\beta$ triggered EMT. Snail is activated by phosphorylation of SMADs (34). Silencing of AMF reduced the TGFBR1 production, resulting in downregulation of Snail (Fig. 3B and C), which was released from the basal stage of suppression of E-cadherin. Funasaka et al (23) showed that the knockdown of AMF induced MET in fibrosarcoma cells, in which Snail was downregulated and E-cadherin was upregulated. Similarly, silencing of AMF/PGI induced MET in osteosarcoma cancer MG-63 cells, in which E-cadherin and GSK-3 $\beta$ were upregulated, while Snail and TGFBR1 were downregulated (13). These accumulated data strongly suggested that knockdown of AMF might regulate the process of EMT through downregulation of TGF $\beta 1$ and Snail to suppression E-cadherin.

Multiple signaling pathways cooperate in the initiation and progression of EMT. For example, Snail cooperates with the transcription regulator ETS1 to activate MAPK signaling pathway and TGFBR1 phosphorylates induced by MAPK pathway. Snail cooperates with other transcription regulators to control gene expression (35). Silencing of AMF in endometrial cancer Ishikawa and HEC-1B cells commonly inhibited MAPK pathway activation (Fig. 4A and B). Moreover, the exogenous PGI/AMF eliminated the effects of MET induced by silencing AMF, while U0126 pretreatment (MAPK pathway inhibitor) abolished this interesting phenomenon. Our results indicate that AMF activates the MAPK pathway and suggest that AMF-mediated activation of MAPK signaling contributes to promotion of EMT in endometrial cancer.

In summary, we demonstrated that AMF expression was high in endometrial cancer tissue and positively related with markers of EMT using tissue microarray. Furthermore, our results with endometrial cancer cells show that AMF silencing suppressed the EMT phenotype and that these effects involved inhibition of the MAPK pathway. Our findings suggest for the first time that AMF/MAPK/EMT system might play a critical role in acquisition of malignant phenotypes in endometrial cancer. Thus, AMF/MAPK/EMT might be a potential therapeutic or prevention strategy for treatment of endometrial cancer.

\section{Acknowledgements}

The study was supported by the National Natural Science Foundation of China (nos. 81172476, 81272885 and 81472427), the Science and Technology Commission of Shanghai Municipality (no. 13JC1404501) and the Doctoral Fund of Ministry of Education of China (no. 20120073110090). 


\section{References}

1. Siegel RL, Miller KD and Jemal A: Cancer statistics, 2015. CA Cancer J Clin 65: 5-29, 2015.

2. Salvesen HB, Haldorsen IS and Trovik J: Markers for individualised therapy in endometrial carcinoma. Lancet Oncol 13: e353-e361, 2012.

3. Sorosky JI: Endometrial cancer. Obstet Gynecol 120: 383-397, 2012.

4. Watanabe H, Takehana K, Date M, Shinozaki T and Raz A: Tumor cell autocrine motility factor is the neuroleukin/phosphohexose isomerase polypeptide. Cancer Res 56: 2960-2963, 1996.

5. Niinaka Y, Paku S, Haga A, Watanabe $\mathrm{H}$ and Raz A: Expression and secretion of neuroleukin/phosphohexose isomerase/maturation factor as autocrine motility factor by tumor cells. Cancer Res 58: 2667-2674, 1998.

6. Kim JW and Dang CV: Multifaceted roles of glycolytic enzymes. Trends Biochem Sci 30: 142-150, 2005.

7. Gurney ME, Apatoff BR, Spear GT, Baumel MJ, Antel JP, Bania MB and Reder AT: Neuroleukin: A lymphokine product of lectin-stimulated T cells. Science 234: 574-581, 1986.

8. Xu W, Seiter K, Feldman E, Ahmed T and Chiao JW: The differentiation and maturation mediator for human myeloid leukemia cells shares homology with neuroleukin or phosphoglucose isomerase. Blood 87: 4502-4506, 1996.

9. Kho DH, Zhang T, Balan V, Wang Y, Ha SW, Xie Y and Raz A: Autocrine motility factor modulates EGF-mediated invasion signaling. Cancer Res 74: 2229-2237, 2014.

10. Kho DH, Nangia-Makker P, Balan V, Hogan V, Tait L, Wang Y and Raz A: Autocrine motility factor promotes HER2 cleavage and signaling in breast cancer cells. Cancer Res 73: 1411-1419, 2013.

11. Bayo J, Fiore E, Aquino JB, Malvicini M, Rizzo M, Peixoto E, Andriani O, Alaniz L, Piccioni F, Bolontrade M, et al: Increased migration of human mesenchymal stromal cells by autocrine motility factor (AMF) resulted in enhanced recruitment towards hepatocellular carcinoma. PLoS One 9: e95171, 2014.

12. Shih WL, Liao MH, Lin PY, Chang CI, Cheng HL, Yu FL and Lee JW: PI 3-kinase/Akt and STAT3 are required for the prevention of TGF-beta-induced Hep3B cell apoptosis by autocrine motility factor/phosphoglucose isomerase. Cancer Lett 290: 223-237, 2010.

13. Niinaka Y, Harada K, Fujimuro M, Oda M, Haga A, Hosoki M, Uzawa N, Arai N, Yamaguchi S, Yamashiro M, et al: Silencing of autocrine motility factor induces mesenchymal-to-epithelial transition and suppression of osteosarcoma pulmonary metastasis. Cancer Res 70: 9483-9493, 2010.

14. Funasaka T, Hogan V and Raz A: Phosphoglucose isomerase/ autocrine motility factor mediates epithelial and mesenchymal phenotype conversions in breast cancer. Cancer Res 69 5349-5356, 2009.

15. Thiery JP and Sleeman JP: Complex networks orchestrate epithelial-mesenchymal transitions. Nat Rev Mol Cell Biol 7: 131-142, 2006.

16. Greenburg G and Hay ED: Epithelia suspended in collagen gels can lose polarity and express characteristics of migrating mesenchymal cells. J Cell Biol 95: 333-339, 1982.

17. Hay ED: The mesenchymal cell, its role in the embryo, and the remarkable signaling mechanisms that create it. Dev Dyn 233: 706-720, 2005 .
18. Lee JM, Dedhar S, Kalluri R and Thompson EW: The epithelialmesenchymal transition: New insights in signaling, development, and disease. J Cell Biol 172: 973-981, 2006.

19. Thiery JP, Acloque H, Huang RY and Nieto MA: Epithelialmesenchymal transitions in development and disease. Cell 139: 871-890, 2009.

20. De Craene B and Berx G: Regulatory networks defining EMT during cancer initiation and progression. Nat Rev Cancer 13: 97-110, 2013

21. Dong P, Karaayvaz M, Jia N, Kaneuchi M, Hamada J, Watari H, Sudo S, Ju J and Sakuragi N: Mutant p53 gain-of-function induces epithelial-mesenchymal transition through modulation of the miR-130b-ZEB1 axis. Oncogene 32: 3286-3295, 2013.

22. Ahmad A, Aboukameel A, Kong D, Wang Z, Sethi S, Chen W, Sarkar FH and Raz A: Phosphoglucose isomerase/autocrine motility factor mediates epithelial-mesenchymal transition regulated by miR-200 in breast cancer cells. Cancer Res 71: 3400-3409, 2011.

23. Funasaka T, Hu H, Yanagawa T, Hogan V and Raz A: Downregulation of phosphoglucose isomerase/autocrine motility factor results in mesenchymal-to-epithelial transition of human lung fibrosarcoma cells. Cancer Res 67: 4236-4243, 2007.

24. Kyo S, Sakaguchi J, Ohno S, Mizumoto Y, Maida Y, Hashimoto M, Nakamura M, Takakura M, Nakajima M and Masutomi K: High Twist expression is involved in infiltrative endometrial cancer and affects patient survival. Hum Pathol 37: 431-438, 2006.

25. Massagué J: TGF $\beta$ signalling in context. Nat Rev Mol Cell Biol 13: 616-630, 2012

26. Cano A, Pérez-Moreno MA, Rodrigo I, Locascio A, Blanco MJ, del Barrio MG, Portillo F and Nieto MA: The transcription factor snail controls epithelial-mesenchymal transitions by repressing E-cadherin expression. Nat Cell Biol 2: 76-83, 2000.

27. Subarsky P and Hill RP: The hypoxic tumour microenvironment and metastatic progression. Clin Exp Metastasis 20: 237-250, 2003.

28. Tsutsumi S, Yanagawa T, Shimura T, Fukumori T, Hogan V, Kuwano H and Raz A: Regulation of cell proliferation by autocrine motility factor/phosphoglucose isomerase signaling. J Biol Chem 278: 32165-32172, 2003.

29. Yang J and Weinberg RA: Epithelial-mesenchymal transition: At the crossroads of development and tumor metastasis. Dev Cell 14: 818-829, 2008

30. Peinado H, Olmeda D and Cano A: Snail, Zeb and bHLH factors in tumour progression: An alliance against the epithelial phenotype? Nat Rev Cancer 7: 415-428, 2007.

31. Prindull G and Zipori D: Environmental guidance of normal and tumor cell plasticity: Epithelial mesenchymal transitions as a paradigm. Blood 103: 2892-2899, 2004.

32. Otto T, Birchmeier W, Schmidt U, Hinke A, Schipper J, Rübben H and Raz A: Inverse relation of E-cadherin and autocrine motility factor receptor expression as a prognostic factor in patients with bladder carcinomas. Cancer Res 54: 3120-3123, 1994.

33. Batlle E, Sancho E, Francí C, Domínguez D, Monfar M, Baulida J and García De Herreros A: The transcription factor snail is a repressor of E-cadherin gene expression in epithelial tumour cells. Nat Cell Biol 2: 84-89, 2000.

34. Pickup M, Novitskiy S and Moses HL: The roles of TGF $\beta$ in the tumour microenvironment. Nat Rev Cancer 13: 788-799, 2013.

35. Lamouille S, Xu J and Derynck R: Molecular mechanisms of epithelial-mesenchymal transition. Nat Rev Mol Cell Biol 15: 178-196, 2014. 